\title{
Evolution des populations des champignons endomycorhiziens sur les adventices de quatre sites maraîchers de la région de Maradi au Niger
}

\author{
Adamou HAOUGUI ${ }^{1 *}$, Pabamé Souapibé SOUNIABE ${ }^{2}$, Ali DOUMMA $^{3}$ et \\ Toudou ADAM ${ }^{3}$ \\ ${ }^{1}$ Institut National de la recherche Agronomique du Niger, BP 429 Niamey, Niger. \\ ${ }^{2}$ ITRAD, BP 5400 N'Djaména, Tchad. \\ ${ }^{3}$ Université de Niamey, Faculté des Sciences et Techniques, Niamey, Niger. \\ *Auteur correspondant,Email: ahaougui@yahoo.com
}

\section{RESUME}

Le rôle important que jouent les endomycorhizes dans l'amélioration de la nutrition minérale et la protection des plantes est aujourd'hui largement reconnue. Cependant dans des pays comme le Niger, peu de travaux leur ont été consacrés. Cette étude a été conduite pour évaluer la mycorhization dans les parcelles de poivron pendant la contre-saison afin de connaitre le moment opportun de les récupérer dans le but de les utiliser ultérieurement dans des programmes de développement des cultures maraichères. Des échantillons de sol et de racines ont été prélevés dans 4 sites sur les 9 principales adventices du poivron. Ils ont été analysés au laboratoire et la densité des spores et l'intensité de l'infection ont été déterminées. Ces deux paramètres ont varié d'un site à l'autre et d'une plante à l'autre. Cinq espèces d'endomycorhizes à vésicules et arbuscules ont été identifiées dont 3 appartenant au genre Glomus et 2 au genre Gigaspora. Acanthospermum hispidum et Solanum nigrum ont été les plantes les plus infectées. Sur les 6 mois de l'étude, les plus fortes densités de spores et d'intensité d'infection ont été enregistrées entre décembre et janvier et les plus faibles en mars. (C) 2013 International Formulae Group. All rights reserved

Mots clés : Endomycorhizes, dynamiques des populations, cultures maraichères, Niger.

\section{INTRODUCTION}

Les mycorhizes à vésicules et arbuscules (MVA) sont des associations symbiotiques entre les racines des plantes supérieures et des champignons de la famille des endogonacées. Cette association améliore l'absorption par la plante de l'eau et les éléments minéraux, en particulier le phosphore. Smith et Smith (2011) ont rapporté une augmentation significative de la concentration du phosphore, du potassium et des oligoéléments sur les plants mycorhizés. Il en est de même des observations faites par
Smith et al. (2011) sur plusieurs espèces de champignons mychoriziens inoculés à différentes espèces végétales. Plusieurs autres auteurs ayant travaillé sur cet aspect, ont conclu que les mycorhizes améliorent la nutrition minérale des plantes même dans les sols pauvres (Leye et al., 2009 ; Bolan et al., 2011). La mycorhization permet aussi d'accroître la résistance des plantes aux mircoorganismes pathogènes du sol comme les agents de pourritures racinaires, de fonte de semis (Whipps, 2004; Echairi et al., 2008 ; Tahat et al., 2010) et des nématodes à 
galles du genre Meloidogyne (Ngonkeu et al., 2007 ; Akthar et Siddiqui, 2008 ; Al-Askar et Rashad, 2010). Toutes ces propriétés sur la plantes résultent de l'extension de la surface d'absorption des racines et du volume de sol exploré par le mycélium des champignons mycorhiziens. C'est pourquoi certains auteurs ont préconisé leur utilisation dans la régénération des forêts tropicaux et des sols dégradés des zones semi-arides (Laminou, 2010 ; Alguacil et al., 2011). Ces propriétés ont aussi fait penser à leur utilisation en agriculture pour augmenter les rendements des cultures. Malgré ce rôle important que jouent les mycorhizes dans la production végétale, peu d'intérêt leur est accordé au Niger.

Cette étude porte sur la recherche de champignons mycorhiziens dans des sites de cultures de contre-saison afin de connaitre leur diversité et le moment opportun de les récolter en vue de leur introduction sur les cultures maraichères.

\section{MATERIEL ET METHODES}

\section{Choix des sites et présentation du milieu}

Quatre sites maraichers ont été retenus dans la vallée d'Aguié $\left(13^{\circ} 30^{\prime} 00 \mathrm{~N}, 7^{\circ} 46^{\prime} 65\right.$ E; Altitude $426 \mathrm{~m}$ ), région administrative de Maradi, où exerce un projet de développement des cultures maraîchères, principalement le poivron. La zone prospectée se trouve dans la parie méridionale du Niger à climat sahélosoudanien avec trois saisons: (i) une saison sèche et froide (novembre-mars) avec une température moyenne de $22{ }^{\circ} \mathrm{C}$; (ii) une saison sèche et chaude (avril-mai) avec une température moyenne de $36{ }^{\circ} \mathrm{C}$; (iii) une saison pluvieuse (juin-octobre) avec une température moyenne de $30{ }^{\circ} \mathrm{C}$. La pluviométrie moyenne annuelle varie entre 450 et $500 \mathrm{~mm}$ (Figure 1).

Sur chaque site, les espèces d'adventices les plus dominantes dans les parcelles de poivron ont été échantillonnées (Tableau 1).

\section{L'échantillonnage}

Dans chaque site les prélèvements ont été faits selon la méthode du Zig-zag de Barker (1985). Des échantillons de sol et de fines racines ont été prélevés dans la rhizosphère des plantes à une profondeur de 5 à $20 \mathrm{~cm}$ à l'aide d'un déplantoir. Six échantillons composites ont été prélevés sur chaque espèce végétale et mis chacun dans un sac plastique. Chaque échantillon a été ensuite étiqueté et mis dans un carton très solide. Le tout a été transporté au laboratoire de phtytopathologie-nématologie du Centre Régional de la Recherche Agronomique de Kollo pour analyse.

\section{Extraction des spores d'endomycorhize du sol}

L'extraction des spores a été faite en utilisant la méthode de Gerdemann et Nicolson (1963). Le comptage des spores a été fait dans des coupelles de nématologie à fond quadrillé sous binoculaire.

Des spores de chaque espèces de MVA sont montées entre lame et lamelle avec du polyvinyle alcool au lactophénol pour identification. La densité des spores (nombre de spores par $10 \mathrm{~g}$ de $\mathrm{sol} \mathrm{sec}$ ) à ensuite été déterminée.

Pour observer les structures intramatricielles des M.V.A, les racines ont été colorées de la manière suivante: elles sont trempées dans du $\mathrm{KOH} 10 \%$ puis mises au bain-marie pendant 10 à $15 \mathrm{mn}$. Après avoir versé le $\mathrm{KOH}$, les racines sont trempées dans du $\mathrm{Hcl} 1 \%$ pendant 3 à $4 \mathrm{mn}$. Elles sont ensuite mises dans le trypan bleu au lactophénol pendant $30 \mathrm{mn}$. Puis, vingt fragments de racine de $1 \mathrm{~cm}$ de long chacun ont été montés entre lame et lamelle avec du lactophénol pour observation au microscope. La fréquence et l'intensité d'infection ont été alors déterminées. La fréquence d'infection est le nombre de fragments de $1 \mathrm{~cm}$ contenant des structures mycorhiziennes sur les 20 montés entre lame et lamelle. Alors que l'intensité d'infection (ou taux de colonisation $=\mathrm{TC}$ ), est le pourcentage de surface colonisée par les MVA dans chacun des 20 fragments. Elle est donnée par la formule suivante.

$$
T c=\frac{\text { taux moyen d'infection }}{\text { nombre de fragements analysés }}
$$




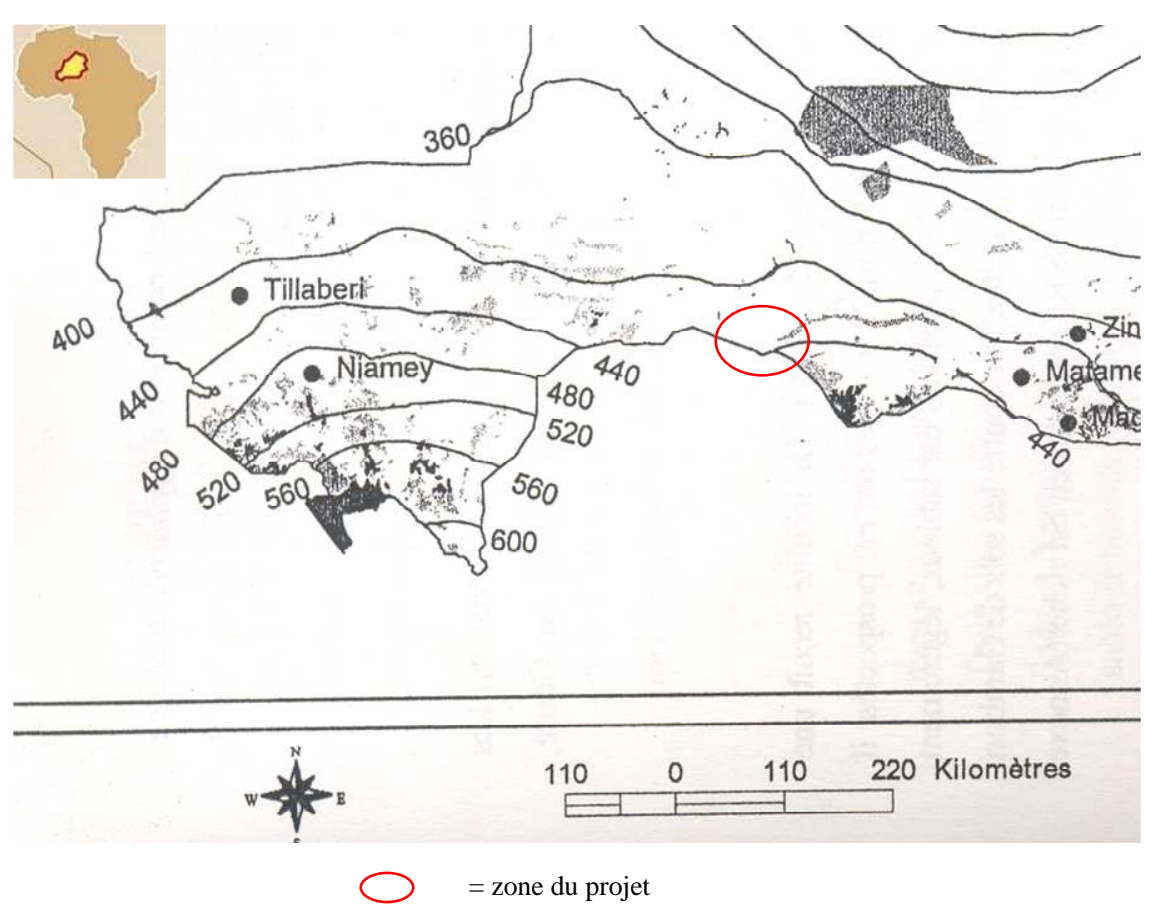

Figure 1: Localisation du site d'étude.

Tableau 1 : Espèces végétales échantillonnées pour l'étude de la mycorhization des sites.

\begin{tabular}{llll}
\hline Site 1 & Site 2 & Site 3 & Site 4 \\
\hline Cenchrus biflorus & Cynodon dactylon & Acanthospermum hispidum & Corchorus olutorus \\
Pennisetum pedicillatum & $\begin{array}{l}\text { Amaranthus hybridus } \\
\text { Portulaca oleracea }\end{array}$ & Solanum nigrum & Cleome viscosa \\
& & \\
\hline
\end{tabular}

\section{RESULTATS}

Les espèces d'endomycorhizes identifiées

Les espèces de M.V.A. rencontrées figurent dans le Tableau 2. Il s'agit de Glomus moseae, Glomus aggregatum, Glomus sp. , Gigaspora heterogama et Gigaspora sp. Les résultats montrent que la fréquence et la densité d'une espèce mycorhizienne varient d'un site à l'autre.

Glomus mosseae est présent sur tous les 4 sites alors que les autres espèces sont cantonnées à un seul site. Glomus sp n'est apparu qu'en décembre dans le site 2 alors que les autres espèces ont été rencontrées durant toute la période d'étude. Gisgapora spp n'ont été retrouvées que sur le site 3. Les espèces dominantes sont $G$. aggregatum sur le site 1 et Glomus moseae sur les autres. Mais en juin la densité de Glomus sp sur Portulaca oleracea du site 2 est plus importante. La fréquence moyenne d'apparition des espèces de M.V.A. varie de 16,6 à 100\% .

\section{Densité des spores par sites}

La Figure 2 montre la densité moyenne des spores par site. La densité moyenne générale de spores obtenue est de 28,48 spores/10 g de sol. Les sites 1 et 2 sont les plus infestés avec des densités de plus de 3,5 fois supérieures à celles des sites 3 et 4 . La Figure 2 montre que la période décembrejanvier est le moment de plus forte production de spores et mars, celui de plus faibles densité de spores. Le pic de production de spores est variable d'un site à l'autre. Pour les sites 1 et 
4, il se situe en janvier alors qu'il est obtenu en décembre sur les sites 2 et 3 .

Les densités des spores dans la rhizosphère des différentes plantes échantillonnées sur les 4 sites sont présentées dans le Tableau 3. Cynodon dactylon, sur le site 2, présente la plus forte densité de spores et Cleome viscosa sur le site 4 a la densité la plus faible.

Parmi les plantes du site 1, Cenchrus biflorus présente en moyenne deux fois plus de spores que Pennisetum pedicillatum. La densité maximale est atteinte en janvier et février sur la première espèce alors qu'elle a été enregistrée sur la seconde dès le mois d'octobre. Sur les deux plantes la densité de spores a diminué au mois de mars.

Sur le site 2, Cynodon dactylon présente une densité dix fois supérieure à celle de Amaranthus hybridus et 4 fois celle de Portulaca oleracea. Les pics de production de spores sur ces plantes se situent respectivement en décembre, janvier et février. Les plantes des sites 3 et 4 sont les moins mycorhizées. Sur le site 3, les deux plantes sont toutes faiblement mycorhizées et ont pratiquement les mêmes densités de spores avec des pics de production de spores situés en décembre pour Acanthospermum hispidum et en janvier pour Solanum nigrum. Les plantes du site 4 (Corchorus olitorus et Cleome viscosa) ont aussi des densités de spores relativement faibles. Leurs pics de production se situent en janvier.

\section{Fréquence et Intensité de l'infection par site}

Les résultats de l'analyse de la mycorhization des racines sont consignés dans la Figure 3 et le Tableau 4. Les racines des plantes du site 3 possèdent les plus grandes fréquences et intensités de mycorhization et celles du site 4 les plus faibles. La fréquence moyenne de mycorhization des racines, tous sites confondus, est de $51,16 \%$ alors que l'intensité moyenne de l'infection est de $37,40 \%$.

Le Tableau 4 montre que la fréquence a évolué de $64 \%$ en novembre à $37,50 \%$ en février. En moyenne, la fréquence de mycorhization est plus forte en novembre et décembre et plus faible en février et mars. Le Tableau 4 montre que la plus forte intensité est obtenue en novembre et la plus faible en janvier. Les pics de mycorhization des racines sont obtenus entre novembre (sites 1 et 4 ) et décembre (sites 2 et 3 ). La Figure 4 montre que le site 3 est le plus infecté et le site 4 présentant la plus faible infectuosité. La Figure 5 montre les intensités d'infection sur les plantes des différents sites prospectés. Il en ressort que toutes les plantes sont infectées mais à de degré différents. Ainsi, les plantes $\mathrm{du}$ site 3 (Acanthospermum hispidum et Solanum nigrum) ont été les plus mycorhizées avec des intensités deux fois et demie supérieures à celles des plantes du site 4 . Les plantes des sites 1 et 2 ont eu une mycorhization moyenne. Cependant, Cynodon dactylon sur le site 2 a présenté le plus faible taux d'infection malgré la plus grande densité de spores trouvées dans sa rhizosphère. Son intensité d'infection est de huit fois inférieure à celle des plantes du site 1 , plus de 4 et 3 fois inférieure celles des plantes des sites 1 et 4 respectivement.

Sur tous les sites la fréquence de mycorhization a été relativement importante avec une moyenne générale de $50 \%$. Sur les sites 1,2 et 4 , toutes les plantes ont une fréquence de mycorhization supérieure à $40 \%$ (Figure 4). Les plantes les plus fréquemment infectées sont celle du site 3 où la fréquence de mycorhiozation moyenne dépasse $70 \%$ (Figure 5).

Le Tableau 5 montre l'évolution de l'infection et de la fréquence d'infection des différentes plantes durant la saison des cultures. En moyenne, la période de novembre-décembre est plus favorable à la colonisation des racines que les autres parties de la saison. Entre janvier et mars, ces deux paramètres sont restés quasi stationnaires. La plupart des plantes ont eu leur pic d'infection entre novembre et décembre à l'exception de Cenchrus biflorus sur le site 1, qui a été plus infecté en octobre. 


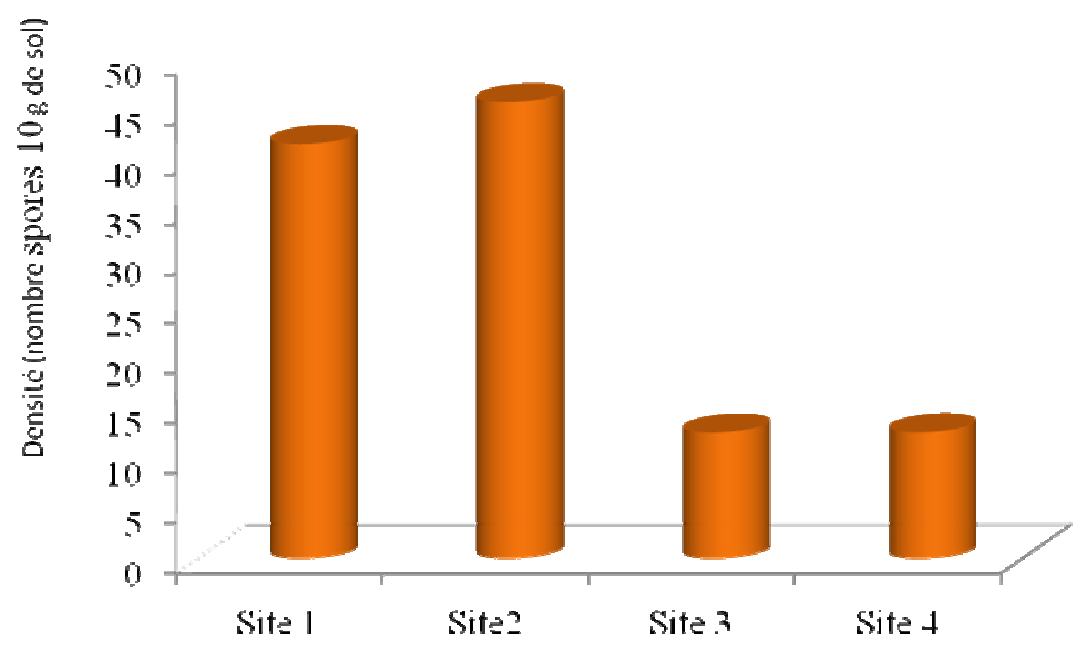

Figure 2: Densités moyenne des spores d'endomycorhizes dans le sol par site.

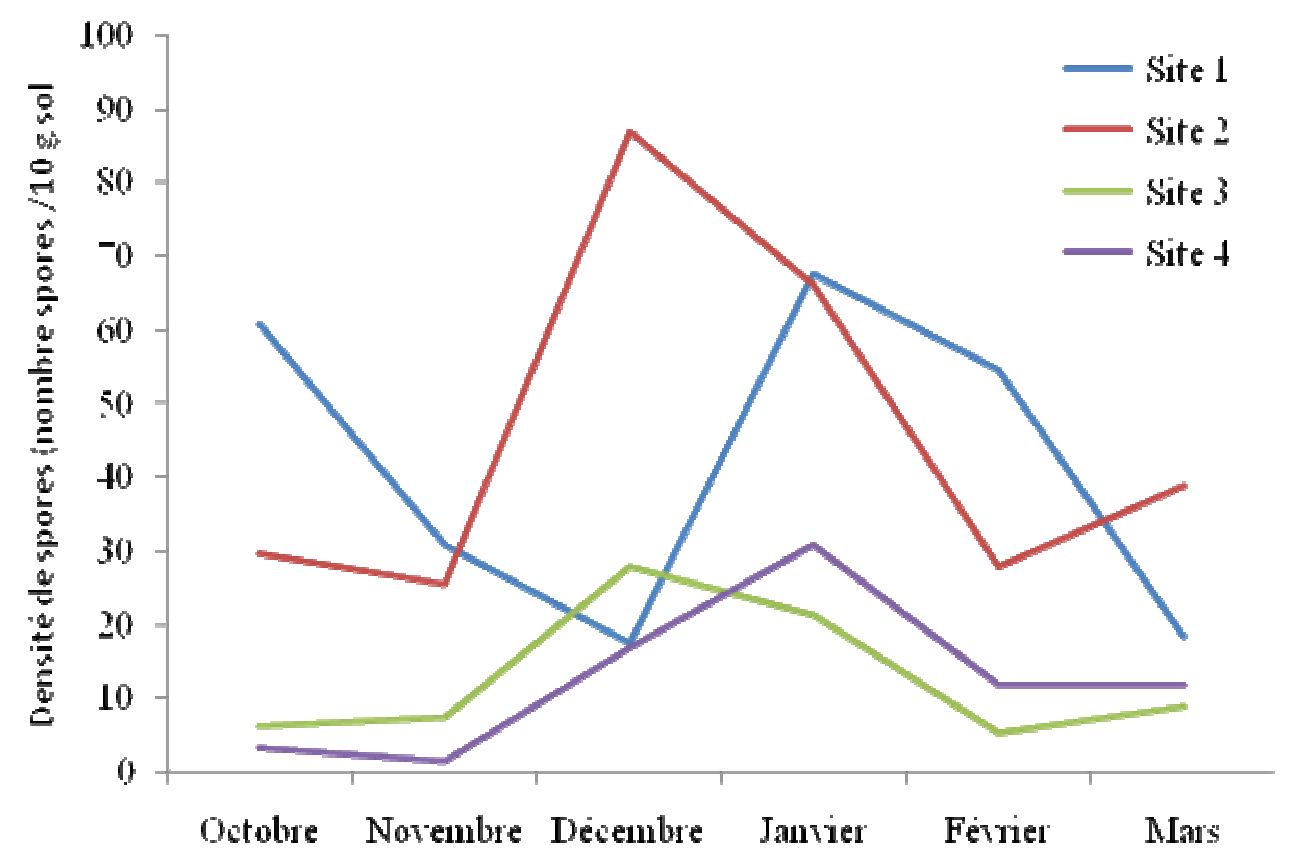

Figure 3: Evolution des densités des spores d'endomycorhizes dans le sol sur les sites. 


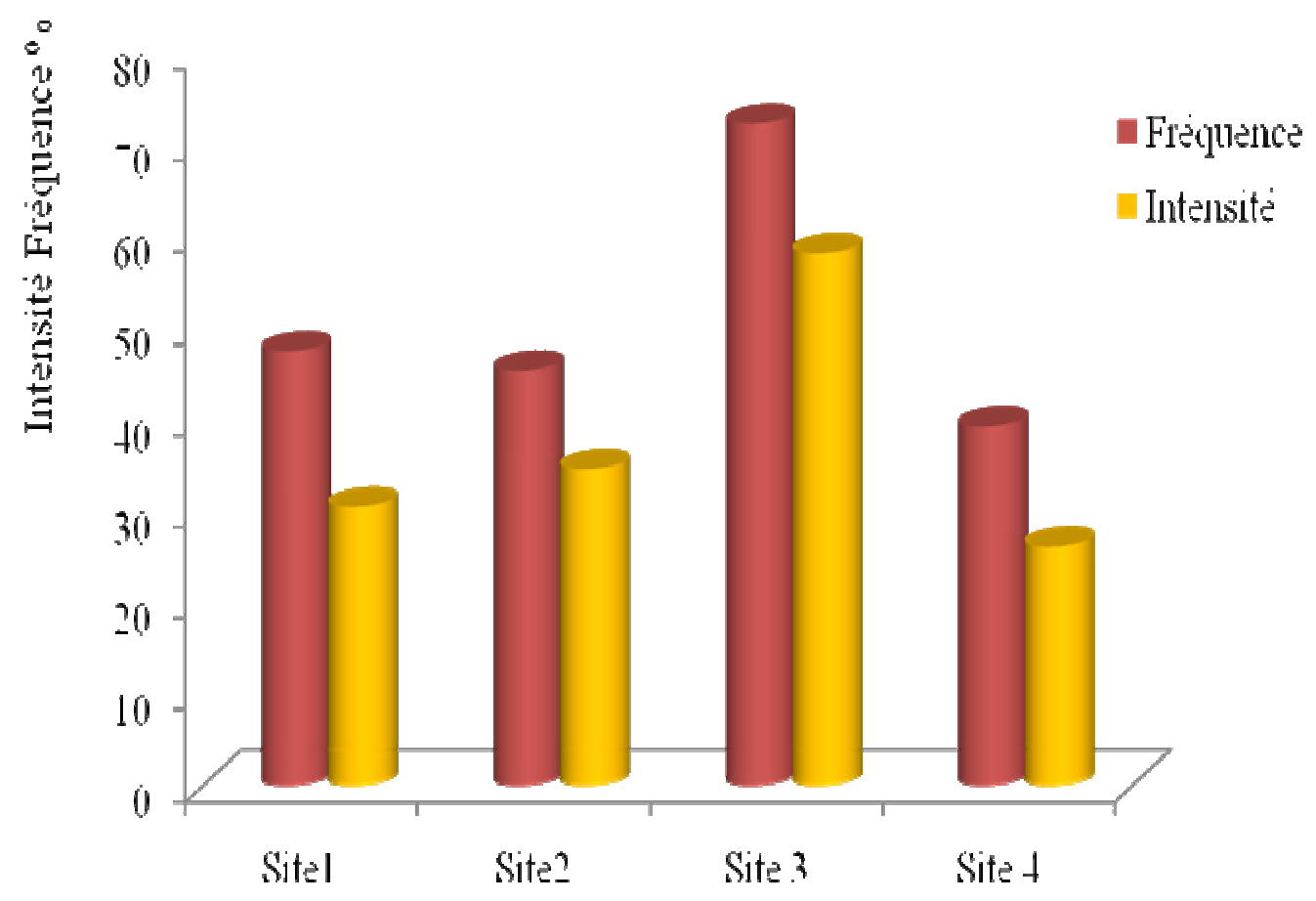

Figure 4: Fréquence et Intensité de mycorhization des racines des différents sites.

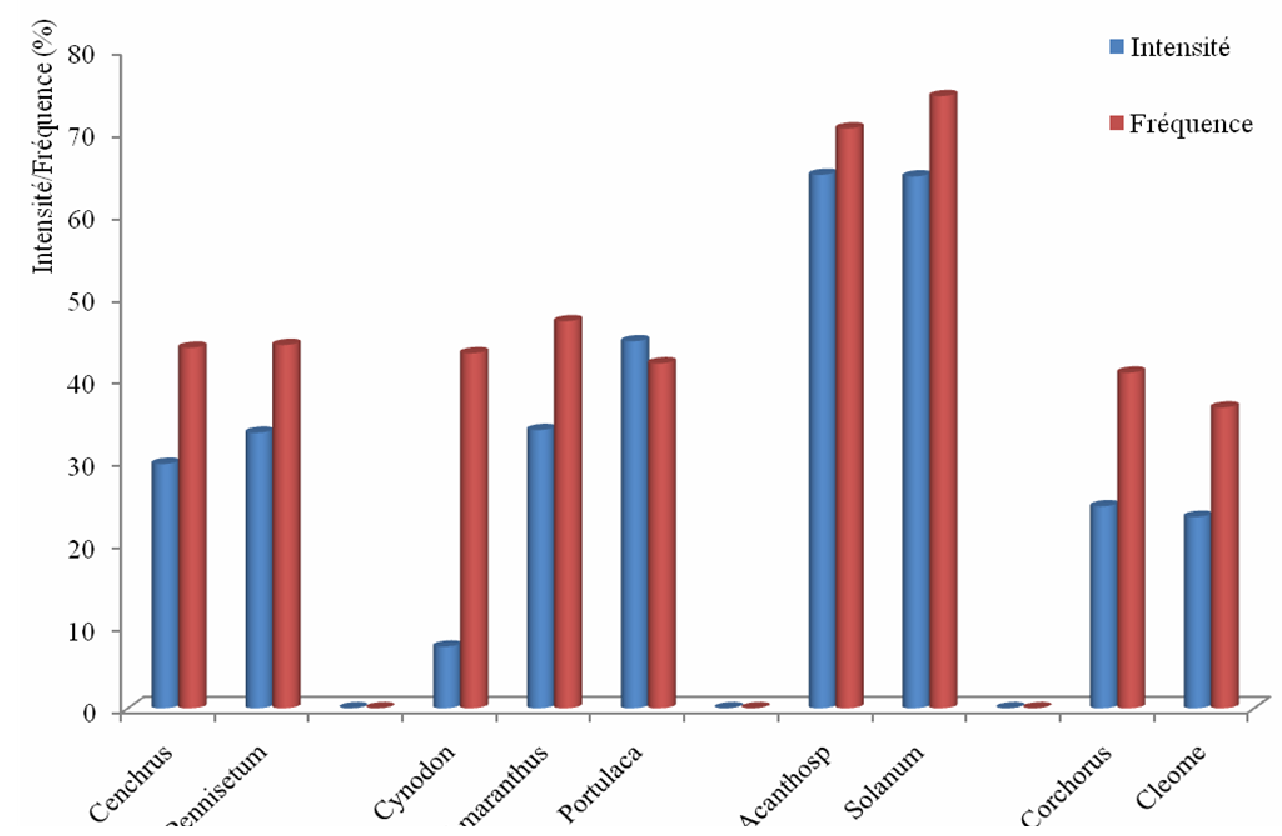

Figure 5: Intensités et fréquence d'infection sur les plantes. 
Tableau 2: Densité des spores et fréquence d'apparition des différentes espèces de mycorhizes dans les échantillons de sol.

\begin{tabular}{|c|c|c|c|c|c|c|c|c|c|c|c|c|}
\hline \multirow[t]{2}{*}{ Sites } & \multicolumn{2}{|c|}{ Oct. } & \multicolumn{2}{|c|}{ Nov. } & \multicolumn{2}{|c|}{ Déc. } & \multicolumn{2}{|c|}{ Jan. } & \multicolumn{2}{|c|}{ Fév. } & \multicolumn{2}{|c|}{ Mars } \\
\hline & D & FA & $\mathbf{D}$ & FA & D & FA & D & FA & D & FA & D & FA \\
\hline \multicolumn{13}{|l|}{ SITE 1: } \\
\hline Glomus aggregatum & 59,5 & 100 & 27,8 & 100 & 15,4 & 100 & 64,8 & 100 & 41,87 & 100 & 31,10 & 87 \\
\hline G. mosseae & 1,6 & 50 & 3,3 & 66,6 & 2,0 & 58,3 & 2,4 & 74,9 & 1,30 & 52 & 1,44 & 41 \\
\hline \multicolumn{13}{|l|}{ SITE 2} \\
\hline G. mosseae & 44,4 & 100 & 38,7 & 91,6 & 80,7 & 88,8 & 173,8 & 100 & 84,4 & 78 & 30,70 & 68 \\
\hline Glomus sp. & - & - & - & - & 6,2 & 66,6 & 3,3 & 77,7 & 2,60 & 56 & 1,80 & 51 \\
\hline \multicolumn{13}{|l|}{ SITE 3 : } \\
\hline G. mosseae & 5,0 & 75 & 5,8 & 75 & 24,6 & 100 & 31,64 & 100 & 20,06 & 75 & 3,91 & 58 \\
\hline Giguspora sp. & 1,2 & 43,1 & 0,6 & 83,3 & 1,1 & 91,6 & 3,63 & 100 & 1,22 & 59 & 0,30 & 21 \\
\hline G. heterogama & 1,5 & 83,3 & 0,7 & 91,6 & 1,6 & 66,6 & 2,08 & 100 & 0,90 & 71 & 0,47 & 67 \\
\hline \multicolumn{13}{|l|}{ SITE 4: } \\
\hline G. mosse & 3,3 & 54,1 & 1,4 & 41,6 & 16,7 & 100 & 21,59 & 83 & 12,04 & 42,7 & 2,03 & 35 \\
\hline
\end{tabular}

\section{DISCUSSION}

Les résultats de cette étude montrent clairement la présence des endomycorhizes à vésicules et arbuscules dans le sol de la rhizosphère et dans les racines de toutes les plantes adventices échantillonnées sur les 4 sites prospectés. Deux genres de ces champignons ont été trouvés, Glomus et Gigaspora. Ces deux genres ont déjà été rencontrés dans des études antérieures entreprises par Ibrahim et al. (1995) et Laminou (2010) sur la mycorhization des plantes au Niger. Le genre Glomus a été présent sur tous les sites prospectés. Il a une large distribution au Niger car toutes les études antérieures effectuées dans ce pays sur les endomycorhizes ont mis en évidence sa présence dans la quasi-totalité des échantillons (Clark et Zeto, 2000; Laminou, 2010). Plusieurs travaux ont démontré la présence des endomycorizes (Glomus en général) dans la rhizosphère de plantes d'autres régions chaudes du globe (Bousselmame et al., 2002 ; Zhao et al., 2003; Laminou, 2010). Dans une étude conduite par Jamil et al. (2002) dans les régions arides et semi-arides de Jordanie, 7 espèces du genre Glomus ont été trouvées. Dans les régions humides du Cameroun et de l'Afrique centrale, Ngonkeu et al. (2007) ont trouvé que Glomus est le plus fréquent sur les 5 genres qu'ils ont trouvés. Schwarzott et al. (2011) ont, dans une étude phyllogénétique récente, conclu que c'est le genre le plus largement répandu à travers les tous écosystèmes terrestres et que cette plasticité s'explique par sa grande hétérogénéité génétique car il peut, à lui seul, constituer une famille.

La présence des MVA sur toutes les plantes et sur tous les sites montrent qu'il n'y a pas de spécificité d'infection. Sur tous les sites, la densité des spores est relativement faible avec des taux infection moyen inférieur à $40 \%$. Cela pourrait s'expliquer par la perturbation des milieux due à l'usage des fumures phosphatées. En effet, les fortes teneurs en NPK inhibent le développement des mycorhizes (Muthukumar et Udaiyan, 2000 Muthukumar et Udaiyan, 2002 ; Turk et al., 2006). Or, sur tous nos sites, 
A. HAOUGUI et al. / Int. J. Biol. Chem. Sci. 7(2): 554-565, 2013

Tableau 3: Densités de spores d'endomycorhizes du sol par plante.

\begin{tabular}{|c|c|c|c|c|c|c|c|c|c|c|}
\hline & \multicolumn{2}{|c|}{ Site 1} & \multicolumn{3}{|c|}{ Site 2} & \multicolumn{2}{|c|}{ Site 3} & \multicolumn{2}{|c|}{ Site 4} & \multirow[t]{2}{*}{ Moyen } \\
\hline & CB & $\mathbf{P P}$ & CD & $\mathbf{A S}$ & PO & AH & SN & $\mathrm{CO}$ & $\mathrm{CV}$ & \\
\hline Oct. & 73,10 & 48,70 & 85,50 & 3,40 & - & 11,00 & 1,10 & 0,60 & 6,00 & 25,49 \\
\hline Nov. & 30,10 & 31,70 & 64,30 & 12,10 & - & 10,50 & 4,10 & 1,60 & 1,30 & 17,30 \\
\hline Déc. & 13,60 & 21,20 & 240,60 & 15,50 & 4,80 & 17,50 & 37,80 & 21,30 & 12,10 & 42,71 \\
\hline Janvier & 34,00 & 101,30 & 157,80 & 21,10 & 19,20 & 22,60 & 19,60 & 43,60 & 17,80 & 48,56 \\
\hline Février & 6,90 & 102,20 & 17,50 & 1,50 & 64,20 & 4,00 & 6,20 & 18,80 & 5,00 & 25,14 \\
\hline Mars & 4,80 & 31,50 & 43,60 & 10,00 & 63,00 & 11,80 & 5,30 & 12,10 & 11,60 & 21,52 \\
\hline Moy. & 27,08 & 56,10 & 101,55 & 10,60 & 25,20 & 12,90 & 12,35 & 16,33 & 8,97 & 30,12 \\
\hline
\end{tabular}

$\mathrm{CB}=$ Cenchrus biflorus $; \mathrm{PP}=$ Pennisetum pedicillatum $; \mathrm{CD}=$ Cynodon dactylon $; \mathrm{AS}=$ Acanthospermum hispidum $; \mathrm{PO}=$ Portulaca oleracea $; \mathrm{AH}=$ Amaranthus hybridus $;$

$\mathrm{SN}=$ Solanum nigrum $; \mathrm{CO}=$ Corchorus olitorus $; \mathrm{CV}=$ Cleome viscosa

Tableau 4: Evolution de la fréquence et de l'intensité d'infection par site.

\begin{tabular}{|c|c|c|c|c|c|c|c|c|c|c|}
\hline \multirow[t]{2}{*}{ Sites/Mois } & \multicolumn{2}{|c|}{ SITE 1} & \multicolumn{2}{|c|}{ SITE 2} & \multicolumn{2}{|c|}{ SITE 3} & \multicolumn{2}{|c|}{ SITE 4} & \multicolumn{2}{|c|}{ Moyenne } \\
\hline & $\mathrm{F}$ & I & $\mathrm{F}$ & I & $\mathrm{F}$ & I & $\mathrm{F}$ & I & $\mathrm{F}$ & I \\
\hline Octobre & 60,7 & 42 & 58 & 36,3 & 71,6 & 53,2 & 33,3 & 19,3 & 55,9 & 37,7 \\
\hline Novembre & 70,5 & 54,5 & 53,3 & 39,2 & 84,4 & 73,2 & 47,5 & 43,5 & 63,925 & 52,60 \\
\hline Décembre & 43,3 & 19,4 & 53 & 39,7 & 92,5 & 78,2 & 65,4 & 32,6 & 63,55 & 42,475 \\
\hline Janvier & 44,1 & 21,7 & 30,6 & 18 & 77,5 & 63,4 & 35 & 15,8 & 46,8 & 29,725 \\
\hline Février & 32,9 & 25,1 & 37,7 & 36,2 & 49,7 & 60,5 & 29,7 & 21 & 37,5 & 35,70 \\
\hline Mars & 33,7 & 20,4 & 39,9 & 38,5 & 58,7 & 21 & 25 & 25 & 39,325 & 26,225 \\
\hline Moyenne & 47,53 & 30,52 & 45,42 & 34,65 & 72,40 & 58,25 & 39,32 & 26,20 & 51,16 & 37,40 \\
\hline
\end{tabular}


A. HAOUGUI et al. / Int. J. Biol. Chem. Sci. 7(2): 554-565, 2013

Tableau 5: Evolution de la fréquence et de l'intensité d'infection par plante.

\begin{tabular}{|c|c|c|c|c|c|c|c|c|c|c|c|c|}
\hline \multirow[t]{2}{*}{ Sites } & \multicolumn{2}{|c|}{ Octobre } & \multicolumn{2}{|c|}{ Nov. } & \multicolumn{2}{|c|}{ Déc. } & \multicolumn{2}{|c|}{ Janv. } & \multicolumn{2}{|c|}{ Février } & \multicolumn{2}{|c|}{ Mars } \\
\hline & II & FI & II & FI & II & FI & II & FI & II & FI & II & FI \\
\hline \multicolumn{13}{|l|}{ SITE 1} \\
\hline Cenchrus biflorus & 66,4 & 82,5 & 42,4 & 61,5 & 1,4 & 23,3 & 16,3 & 16,3 & 35,0 & 22,3 & 16,6 & 26,6 \\
\hline Pennisetum pedicillatum & 17,7 & 39,1 & 66,6 & 80 & 37,4 & 63,3 & 27,1 & 53,3 & 27,8 & 44,5 & 24,2 & 48,8 \\
\hline \multicolumn{13}{|l|}{ SITE 2} \\
\hline Cynodon dactylon & 27,7 & 47,0 & 57,5 & 72,5 & 36,7 & 49,1 & 13,3 & 21,1 & 12,1 & 37,7 & 17,6 & 30,8 \\
\hline Amaranthus spinosus & 44,9 & 69,1 & 21,0 & 34,1 & 60,1 & 75,0 & 25,1 & 39,1 & 24,8 & 34,8 & 26,9 & 30,0 \\
\hline Portulaca oleracea & - & & & - & 21,9 & 35,0 & 15,6 & 31,6 & 72,3 & $57 ; 8$ & 68,5 & 59,1 \\
\hline \multicolumn{13}{|l|}{ SITE 3} \\
\hline A. hispidum & 45,3 & 63,3 & 74,7 & 85,7 & 80,8 & 93,3 & 63,6 & 75,8 & 59,0 & 51,2 & 65,5 & 53,4 \\
\hline Solanum nigrum & 61,2 & 80,0 & 64,3 & 83,2 & 76,8 & 91,6 & 63,0 & 79,1 & 61,8 & 48,3 & 60,6 & 64,1 \\
\hline \multicolumn{13}{|l|}{ SITE 4} \\
\hline Corchorus olitorus & 18 & 25,25 & 55,5 & 45,0 & 35,0 & 74,1 & 16,5 & 36,6 & 22,9 & & 22,2 & 20,0 \\
\hline Cleome viscosa & 20,3 & 22,40 & 31,5 & 50,0 & $30 ; 3$ & 56,6 & 15,3 & 33,3 & 19,2 & & 23,2 & 30,0 \\
\hline
\end{tabular}


les producteurs utilisent les engrais minéraux sous forme de NPK (15 15 15) ou de diammonium phosphate $18 \% \mathrm{~N}$ et $46 \% \mathrm{P}$ ) sans aucun contrôle. L'application de certaines pratiques comme l'utilisation des pesticides, en particulier les fongicides, perturbe aussi la mycorhization des plantes comme l'ont constaté Wilson et Williamson (2008) en étudiant l'effet du bénomyl sur la colonisation des racines de Andropogon gerardii par Glomus, Entomphtora, Acaulospora et Scutellospora. . Dans notre étude, la densité moyenne de spores était de près de 30 spores pour 10 grammes de sol. Ce résultats est en concordance avec ceux obtenus dans la partie ouest du pays par Ibrahim et al. (1995).

Les plus fortes densités de populations ont été obtenues entre décembre et janvier et les plus faibles en mars. La saisonnalité des MVA résulte de l'influence des conditions climatiques - température et taux d'humidité du sol- (Ibrahim et al., 1995) et du stade végétatif des plantes. Hatimi et Tahrouch (2007) ont démontré que la mycorhization dépend de l'évolution des niveaux d'éléments nutritifs dans le sol et ont a montré que la production des spores est importante pendant la floraison des plantes et décroit en fin de saison quand les plantes sont en fin de cycle avec le changement de physiologie des racines.

Dans notre cas, le mois de mars est très chaud et coïncide avec la fin des cultures de poivron dans la région. Les producteurs cessent d'irriguer car les plantes et les mauvaises herbes sont en sénescence. Donc le taux d'humidité baisse dans le sol et les racines sont quasiment mortes et dans certains cas, en décomposition très avancée.

\section{Conclusion}

Les données obtenues lors de cette étude réalisée sur les sites de production de poivron de la région de Maradi permettent de conclure que toutes les plantes adventices du poivron échantillonnées sont mycorhizées sur tous les sites et que le moment propice de récupérer les endomycorhizes est la période de décembre à janvier. Vu le rôle important joué par les champignons endomycorhiziens dans l'amélioration de l'alimentation hydrique et minérale des plantes, leur utilisation en agriculture peut aider à accroitre la production des cultures maraichères comme le poivron et à les protéger contre les agents de maladies. Il faudrait alors chercher les conditions optimales de leur multiplication dans le sol sur des plantes locales à forte capacité de mycorhization, comme Solanum nigrum et Acanthospermum hispidum.

\section{REMERCIEMENTS}

Les auteurs remercient le Directeur Général de l'Institut National de la Recherche Agronomique (INRAN) ainsi que le Coordonateur du Projet KKM de Maradi pour leur aide financière combien importante dans la réalisation de cette étude.

\section{REFERENCES}

Akthar MS, Siddiqui ZA. 2008. Arbuscular mycorrhizal fungi as potential bioprotectants against plant pathogens. In Mycorrhiza: Sustainable Agriculture and Forestry, Siddiqui ZA, Akhtar MS, Futai F (eds). Springer Dordrecht: Netherlands; 61- 97.

Al-Askar AA, Rashad YM. 2010. Arbuscular Mycorhizal fungi: a biocontrol agent against common bean Fusarium root rot disease. Plant Pathology Journal, 9: 3138.

Alguacil MDM, Torrecillas E, Kohler J, Roldan A. 2011. A molecular approach to ascertain the success of in situ AM fungi inoculation in the revegetation of a semiarid degraded land. Sci. Total Envirn., 15: 2874-2880.

Barker KR. 1985, Sampling nematode communities. In An advanced Treatise on Meloidogyne, Methodology (vol. 2) Barker R, SASSER JN (eds). North 
Carolina State University Graphics, Raleigh: USA, 3-14.

Bolan NS, Robson AD, Barrow NJ. 2011. Effects of vesicular arbuscular mycorrhiza on the availability of ironphosphates to plant. Plant and Soil, 89: 401-410.

Bousselmame F, Kenny L, Achouri M. 2002. Effet des mycorhizes à vésicules et arbuscules sur la croissance et la nutrition de l'arganier (Argania spinosa L.). Actes Inst. Agron Vet., 22: 193-198.

Clark RB, Zeto SK. 2000. Mineral acquisition by arbuscular mycorhizal plants. J. Plant. Nutr., 23: 867-902.

Echairi A, Nouam R, Chaussod R. 2008. Mycorrhizal inoculation of argan trees (Argania spinosa) in nursery conditions. Sécheresse, 4: 277-281.

Gederman JW, Nicolson TH. 1963. Spores of mycorrhizal Endogone extracted from soil by sieving and decanting. Trans. $B r$. Mycol. Soc., 46: 235-244.

Hatimi A, Tarouch S. 2007. Caractérisation chimique, botanique et microbiologique $\mathrm{du}$ sol des dunes littorales du SoussMassa. Biomatec Echo, 2(5): 85-97.

Ibrahim D, Pierart P, Theon D. 1995. Premières observations sur les mycorhizes vésiculo-arbusculaires de la rhizosphère de quelques spermaphytes du Sud-Ouest du Niger. Belgi. J. Bot., 28: 151-164.

Jamil MM, Rushdi H, Malkawi I. 2002. Populations of arbuscular mycorrhizal in semi-arid environment of Jordan as influenced by biotic and abiotic factors. $J$. Arid Envir., 53: 409-417.

Laminou MO. 2010. Fixation des dunes dans le Sud-Est du Niger: évaluation de l'efficacité de la barrière mécanique, espèces ligneuses adaptées et potentialités d'inoculation mycorhizienne. Thèse de doctorat en Sciences agronomiques et ingénierie biologique, Université de Liège, p. 142.

Leye EHM, Ndiaye F, Diallo B, Sarr AS, Diouf M, Diop T. 2009. Effet de la mycorhization sur la croissance et le développement de Jatropha curcas L. Rev Energ. Renouvel., 12(2): 269-278.

Muthukumar T, Udaiyan K. 2000. Influence of organic manures on arbuscular mycorrhizal fungi associated with Vigna unguiculata (L) Walp. In relation to tissue nutrients and soluble carbohydrate in roots under field conditions. Biol. Soil, 31: 114

Muthukumar T, Udaiyan K. 2002. Seasonality of vasicular-arbucular mycorrhizae in sedges in semi-arid tropical grassland. Acta Oecologica, 23: 337-347.

Ngonkeu MEL, Charles T, Amougou A, Dreyfus B, Chaintreuil C, Moulin L, Bena G, Nwaga D, Tondje P. 2007. Diversité moléculaire des mycorhizes à arbuscules des sols acides à toxicité aluminique et manganique de la zone forestière humides du Cameroun. Communication présentée à l'atelier du réseau de chercheurs sur les biotechnologies végétales, l'amélioration des plantes et sécurité alimentaire (BIOVEG) tenu du 17-19 décembre 2007 à Yaoundé, CRESA Forêt-Bois.

Schwarzott D, Walker C, Chubler S. 2011. Glomus, the Largest Genus of the arbuscular mycorrhizal fungi (Glomales), ss nonmonophyletic. Molecul. Phyllogen. Evol., 21(2): 190-197.

Smith SE, Jacobsen I, Gronlund M, Smith A. 2011. Roles of arbuscular mycorrhizas in plant phosphorus nutrition: Interactions between pathways of phosphorus uptake in arbuscular mycorrhizal roots have important implications for understanding and manipulating plant phosphorus acquisition. Plant Physiol., 156: 10501057. 
Smith SE, Smith FA. 2011. Roles of arbuscular mycorrhizas in plant nutrition and growth: new paradigms fromcellular toecosystem scales. Ann. Rev. Plant Biol., 63: 227-250.

Tahat MM, Sijam K, Othman R. 2006. Mycorrhizal fungi as biocontrol agent. Plant Pathol. Journ., 9: 198-2007.

Turk MA, Assaf TA, Mameed KM, Al-Tawha AM. 2006. Significance of Mycorrhizae. World J. Agric., 2(1): 16-20.
Whipps JM. 2004. Prospect and limitations for mycorrhizas in biocontrol of root pathogens. Canad. Journ. Botan., 83: 1198- 1227.

Wilson GWT, Williamson MM. 2008. Topsin-M: the new benomyl for mycorrhizal suppression experiments. Mycologia, 100(4): 548-554.

Zhao W, Zhibin H, Zhigang L. 2003. Biological mechanism of sandy desertification in grassland reclamation area in North China. Advances Earth Sc., 18(2): 257-263. 